\title{
Detection of Three Sialylated Tumor-Associated Antigens in Malignant Serous Effusions
}

\author{
JUN-ICHI KADOTA \\ The Second Department of Internal Medicine, Nagasaki \\ University School of Medicine, Nagasaki 852
}

\begin{abstract}
Kadota, J. Detection of Three Sialylated Tumor-Associated Antigens in Malignant Serous Effusions. Tohoku J. Exp. Med., 1990, 161 (3), 171-183 One hundred thirty-seven fluid samples from patients with malignant diseases, including 58 with lung cancer and 36 with gastric carcinoma, were screened for three tumor-associated carbohydrate antigens. The antigens tested were sialylated Lewis $^{\times}$(SLEX) antigen, sialylated SSEA-1 (SLX) antigen, and sialylated Lewis ${ }^{a}$ (CA19-9) antigen. Thirty-four fluid samples from patients with benign diseases including 20 with tuberculous pleurisy were also tested for these three antigens as a control. SLEX antigen had the highest positivity rate among the three antigens both in the pleural effusions of patients with pulmonary adenocarcinoma and for all types of lung cancer $(64.1 \%$ and $46.6 \%$, respectively). In cancers of digestive system, the percentage of SLEX positivity was almost same as that for CA19-9, and higher than that for SLX. A combination assay using the three antigens resulted in an increased rate of detection of tumor-associated antigens $(68 \%$ of 58 lung cancers including $83 \%$ of 39 adenocarcinomas, as well as $77 \%$ of 63 digestive system cancers including $84 \%$ of 36 gastric carcinomas). Gel filtration of pleural effusions or bile using sephacryl S-1000 showed that these three antigens were eluted in the void volume, indicating a molecular weight of $>2 \times 10^{6}$. Thus, all 3 antigens possibly exist on carrier protein with a high molecular weight. These observations suggest the potential application of these antigens to distinguish between benign and malignant fluid, and to monitor the effects of the treatment of various types of cancer.— tumor marker; sialylated Lewis ${ }^{\times}$antigen ; malignant effusion
\end{abstract}

Recently, various monoclonal antibodies have been developed to determine tumor-associated antigens in the sera of cancer patients, and have been employed for the diagnosis of malignant disease. CA19-9, the epitope of which is a carbohydrate moiety (Magnani et al. 1982), is a well known useful tumor marker for gastrointestinal malignancies, particularly pancreatic carcinoma. CA125 and DU-PAN-2 are other tumor-associated antigens which are utilized as tumor markers in patients with ovarian cancer (Bast et al. 1981, 1983) and pancreatic carcinoma (Metzgar et al. 1984), respectively. The epitopes of CA125 and DU-PAN-2 are unknown as yet, while that of CA19-9 has been identified as

Received May 28, 1990 ; revision accepted for publication June 27, 1990. 
TABLE 1. Carbohydrate structure of immunodominant epitope of the sialylated forms of the antigens

\begin{tabular}{|c|c|}
\hline Antigen & Structure of immunodominant epitope \\
\hline Sialyl-Lewis ${ }^{\times}$ & $\begin{array}{r}\text { NeuAc } \alpha 2 \rightarrow 3 \text { Gal } \beta 1 \rightarrow 4 \text { GlcNAc } \beta 1 \rightarrow 3 \text { Gal } \beta 1 \rightarrow \\
3 \\
\uparrow \\
\text { Fuc } \alpha 1\end{array}$ \\
\hline Sialyl-Lewis ${ }^{\mathrm{a}}$ & $\begin{array}{r}\text { NeuAc } \alpha 2 \rightarrow 3 \text { Gal } \beta 1 \rightarrow 3 \text { GlcNAc } \beta 1 \rightarrow 3 \text { Gal } \beta 1 \rightarrow \\
4 \\
\text { Fuc } \alpha 1\end{array}$ \\
\hline Sialyl-SSEA-1 & $\begin{array}{r}\text { NeuAc } \alpha 2 \rightarrow 3 \operatorname{Gal} \beta 1 \rightarrow 4 \text { GlcNAc } \beta 1 \rightarrow 3 \text { Gal } \beta 1 \rightarrow 4 \text { GlcNAc } \beta 1 \rightarrow \\
3 \\
\uparrow \\
\text { Fuc } \alpha 1\end{array}$ \\
\hline
\end{tabular}

NeuAc, N-acetylneuraminic acid; Gal, galactose; GlcNAc, N-acetylglucosamine; Fuc, fucose.

sialylated Lewis ${ }^{\text {a }}$ (Magnani et al. 1982). Sialylated Lewis ${ }^{\times}$(SLEX) is recognized by the monoclonal antibody CSLEX1 (Fukushima et al. 1984), a positional isomer of CA19-9 shown in Table 1, and SLEX titers are frequently elevated in the sera of patients with lung cancer, especially pulmonary adenocarcinoma (Hirota et al. $1985 a, b)$.

Sialylated SSEA-1 (SLX) is also shown in Table 1. This is sialylated Lewis ${ }^{\times}$ bound to $\mathrm{i}$ antigen (the precursor of the blood group $\mathrm{ABH} 1$ antigen), and this antigen has also been found to show elevated serum titers in various types of adenocarcinoma, especially pulmonary adenocarcinoma (Kannagi et al. 1986).

The development of pleural effusion or ascites is an important and common finding in the course of many malignant and non-malignant conditions, but distinguishing between the two etiologies can be difficult unless cytological, histological, bacteriological investigations give positive results. Therefore, in the present study we investigated whether the new sialylated tumor-associated antigen SLEX could be used as a diagnostic tool for malignant by a comparison with CA19-9 and SLX.

\section{Materials and Methods}

\section{Materials}

Specimens of 137 malignant effusions, including 70 of malignant ascites, were collected from 58 patients with primary lung cancer (39 adenocarcinomas, 6 small cell carcinomas, 7 squamous cell carcinomas, 3 large cell carcinomas, and 3 others), and from 79 patients with other malignancies. These comprised 36 with gastric carcinoma ( 1 pleural effusion and 35 
ascitic fluid specimens), 11 hepatomas (all ascitic fluid), 7 pancreatic carcinomas (all ascitic fluid), 5 colon cancers (all ascitic fluid), 4 bile duct cancers (all ascitic fluid), 7 ovarian cancers (2 pleural effusions and 5 ascitic fluid specimens), 3 breast cancers (2 pleural effusions and 1 ascitic fluid specimen), and 6 other cancers ( 4 pleural effusions and 2 ascitic fluid specimens).

The fluid samples were considered malignant by cytologic evaluation, and the primary tumors were defined histologically. Benign effusions (29 pleural effusions and 5 ascitic fluid specimens) were obtained from 20 patients with tuberculous pleurisy and 14 patients with other benign diseases ( 4 with liver cirrhosis, 2 with cardiac failure, 2 with pneumothorax, 2 with pneumonia, 2 with ovarian cyst, and 2 others).

\section{Detection of sialylated Lewis ${ }^{\times}$antigen (SLEX)}

A fluorescent enzyme immunosorbent assay (FEIA) was performed by the sandwich method. In brief, CSLEX1 antibody (Fukushima et al. 1984) was added to microfluor "W" plates (Dynatech Laboratories Inc., Alexandria, VA, USA) as the primary antibody, followed by the addition of fluid samples diluted 8-fold with buffer $\mathrm{A}(1 \mathrm{mM} \mathrm{MgCl}, 0.1 \mathrm{M} \mathrm{NaCl}$, $0.5 \% \mathrm{BSA}$, and $0.1 \mathrm{M} \mathrm{NaN}_{2}$ ). After a $24-\mathrm{hr}$ incubation at $4^{\circ} \mathrm{C}$, the wells of the plate were washed with phosphate-buffered saline (PBS, 0.05 M, pH 7.2, GIBCO, Grand Island, NY, USA), and then incubation with the secondary antibody was performed for $2 \mathrm{hr}$ at room temperature. A $\beta$-D-galactosidase binding CSLEX1 (Fuziwara et al. 1988) was employed as the secondary antibody after 250-fold dilution. The absorbance was then measured using a micro FLUOR reader (Dynatech Laboratories Inc.) at an excitation wavelength of $490 \mathrm{~nm}$ and an emission wavelength of $510 \mathrm{~nm}$ after a 40-min incubation with 4methylumbelliferyl $\beta$-D-galactopyranoside as a substrate. The standard antigen was obtained from the culture supernatant of a lung cancer cell line (PC-9, kindly supplied by Dr. Y. Hayata of Tokyo Medical College, Tokyo) according to the following procedure. The culture supernatant was fractionated through Sephacryl S-1000 after 40-fold concentration by a thin channel ultrafiltration system (Amicon Inc., Danvers, MA, USA), and the fractions with higher antigen activity were collected. Protein content was estimated using a protein assay kit (Bio-Rad, Richmond, CA, USA) and the quantity of antigen was expressed in arbitary units based on a comparison with the protein content of the standard ( $1 \mathrm{unit} / \mathrm{ml}$ equalled $8 \mu \mathrm{g} / \mathrm{ml}$ of protein).

\section{Screening for other antigens}

Both CA19-9 and SLX titers were measured using radioimmunoassay kits available commercially (Otsuka Assay Laboratory, Tokushima). The cut-off values for these three antigens were set as the mean \pm 3 s.D. of the titers in the benign fluid samples, i.e., 69 units/ $\mathrm{ml}$ for SLEX, 148 units/ml for SLX, and 31 units/ml for CA19-9.

\section{Fractionation of malignant fluid samples}

The malignant fluid samples were centrifuged at 3,000 rpm for $10 \mathrm{~min}$ and then applied to either a Sephacryl S-300 or S-1000 column $(5 \times 120 \mathrm{~cm})$ equilibrated in PBS $(0.05 \mathrm{M}, \mathrm{pH}$ 7.2). Fractions $(10 \mathrm{ml})$ were collected and were assayed for antigens by the methods described above, and for protein by the absorbance of light at $280 \mathrm{~nm}$.

The statistical significance of differences was determined using Student's $t$-test and the $\chi^{2}$ test. The difference was judged to be significant when a $p$ value was less than 0.05 .

\section{Results}

\section{Benign serous fluid samples}

The mean of the titer of SLX, CA19-9, and SLEX in serous fluid samples obtained from the patients with benign diseases were 57.5 $\pm 30.1,8.5 \pm 7.5$ and 
$27.5 \pm 13.8 \mathrm{U} / \mathrm{ml}$, respectively. There were one $(2.9 \%)$, two $(5.9 \%)$ and two $(5.9 \%)$ false positive results for SLX, CA19-9 and SLEX in these 34 benign fluid samples respectively when the cut-off value was set as the mean +2 s.D. (data not shown), whereas there was only one false positive for SLX and CA19-9 and none for SLEX when the cut-off level was the mean+3s.D. (Fig. 1). We therefore employed the mean +3 s.D. as the cut-off value in this study.

\section{Malignant pleural effusions from patients with lung cancer}

The marker were positive in malignant pleural effusions from all lung cancer patients at the following levels ; $46.6 \%$ for SLEX, $41.4 \%$ for CA19-9, and $32.0 \%$ for SLX (Fig. 1). As also shown in Fig. 1, of 39 patients with lung adenocarcinoma 25 had elevated pleural fluid SLEX levels (64.1\%) including 12 cases where the level was over $1,000 \mathrm{U} / \mathrm{ml}$. There was a statistically significant difference between the patients with benign effusions and this group $(p<0.01$,

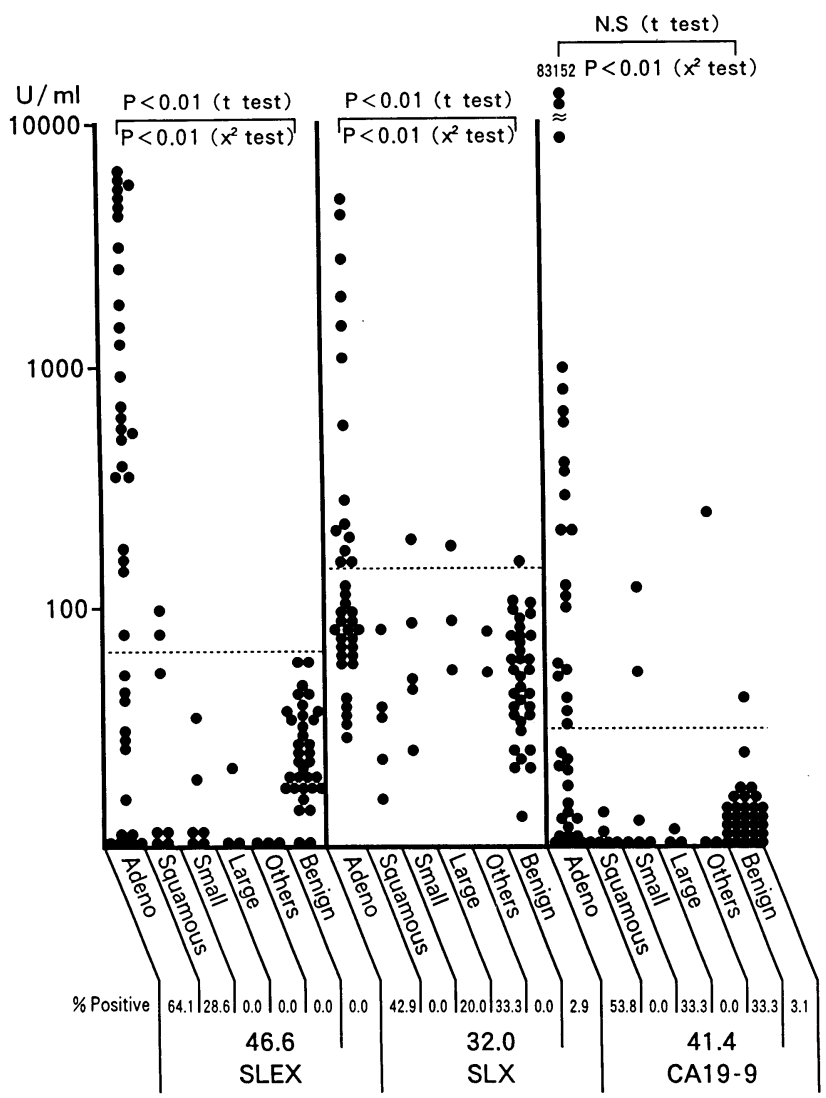

Fig. 1. Levels of the three antigens in the pleural effusions from patients with lung cancer and benign discase. The three antigens were assayed on the same panel of pleural effusions. NS : not significant. 
unpaired Student's $t$-test). CA19-9 and SLX were respectively positive in $53.8 \%$ and $42.9 \%$ of these patients, lower percentage than for SLEX (Fig. 1). A significant difference was also seen for SLX $(p<0.01)$, but not for CA19-9 by the $t$-test, while a significant difference $(p<0.01)$ against the levels in benign disease was shown for all of these antigens by the $\chi^{2}$ test (Fig. 1). As for the patients with other types of lung cancer (Fig. 1), SLEX levels in the pleural fluid were elevated a little over the cut-off value in only two $(28.6 \%)$ of the seven patients with squamous cell carcinoma. CA19-9 and SLX levels were respectively elevated in $33.3 \%$ and $20 \%$ of the patients with small cell carcinoma, and in none and $33.3 \%$ of those with large cell carcinoma. The percentages of SLEX positivity in pulmonary adenocarcinoma or even in all lung cancer were higher than those for CA19-9 and SLX.

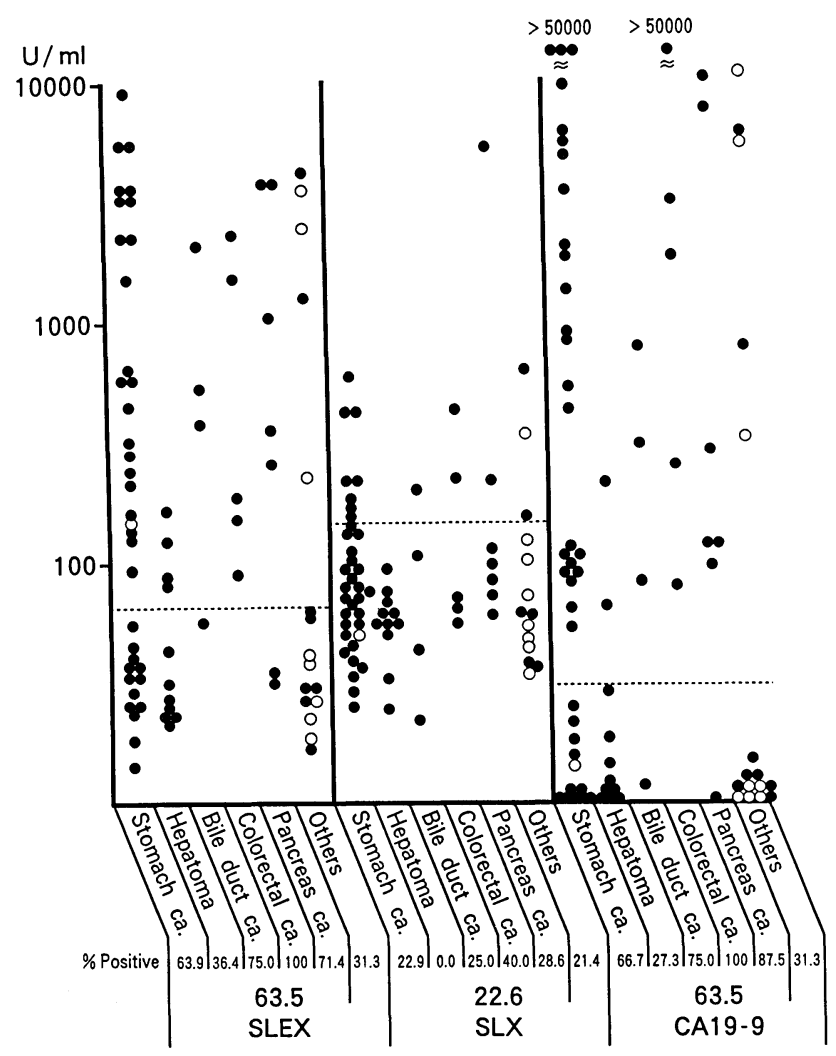

Fig. 2. Levels of the three antigens in the fluid samples from patients with cancer of digestive system. In the malignant fluid samples from other types of cancers, SLEX antigen was positive in ovarian cancer, breast cancer and chronic myelocytic leukemia. CA19-9 was positive for ovarian cancer and malignant lymphoma, and SLX was positive for ovarian cancer and breast cancer. The three antigens were assayed on the same panel of the fluid samples. $\bigcirc$, pleural effusion; $\bullet$, ascites. 


\section{Other malignant fluid specimens}

Fig. 2 shows the levels of the three antigens in malignant fluid specimens from patients with other types of cancer. Forty $(63.5 \%)$ of 63 malignant fluid specimens from patients with cancers of digestive system were positive for SLEX, including $63.9 \%$ of the 36 gastric carcinomas, 3 of 4 bile duct cancers, all 5 of the colorectal cancers, and $71.4 \%$ of the 7 pancreatic carcinomas.

CA19-9 paralleled SLEX in cancers of digestive system, whereas SLX positivity was much lower than that of the other two antigens. There were significant differences in both SLEX and CA19-9 positivity between benign disease and these cancers $(p<0.01$ and $p<0.05$, respectively; unpaired $t$-test), while significant differences were shown for 3 antigens (SLEX, $p<0.01$; CA19-9, $p<0.01$; and SLX, $p<0.05, \chi^{2}$ test). In all other types of cancers, the percentages of positivity were $31.3 \%$ for SLEX, $31.3 \%$ for CA19-9, and $21.4 \%$ for SLX. High positivity rates were observed for SLEX (42.8\%) and CA19-9 (71.4\%) in ovarian cancer fluid specimens while SLX was only positive $14.3 \%$ (data not
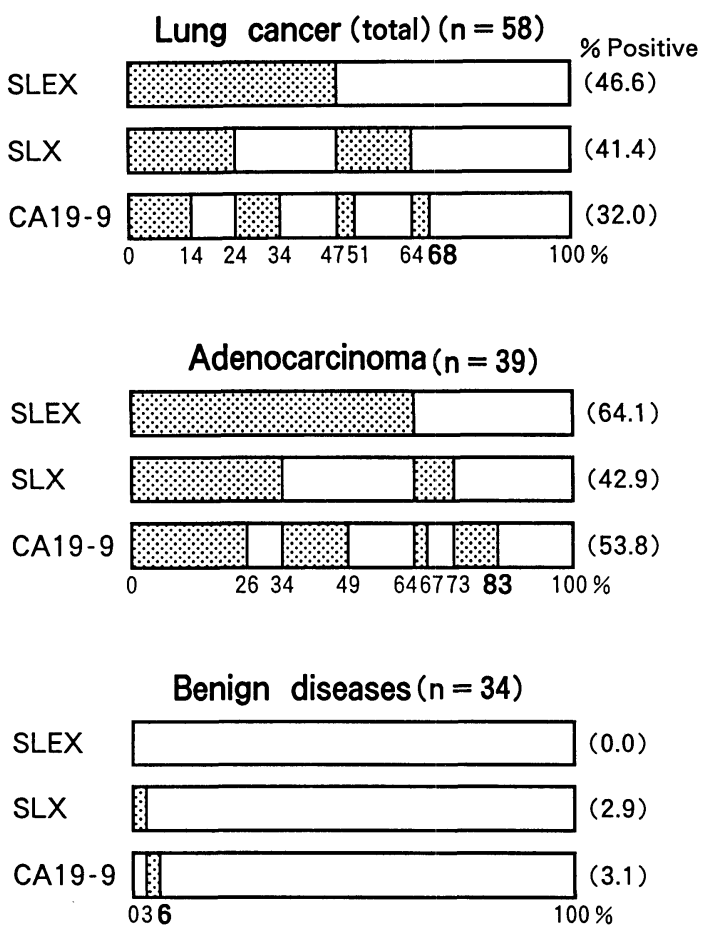

Fig. 3. The combination assay of three antigens in the pleural effusions from 58 patients with lung cancer including 39 adenocarcinomas and in the fluid samples from 34 patients with benign disease. Numbers in parentheses on right side indicate positivity rates in each antigen. Boldface numbers indicate the total percentages of which at least one of three antigens is positive in each group. 
shown).

Correlations between the three antigens and the results of combination assays

No significant correlation was found between any pairs of the three antigens in pleural fluid from patients with pulmonary adenocarcinoma, and the same result was obtained for all lung cancer (data not shown). There was also no significant correlation between any pairs of these three antigens in digestive organ cancers or gastric carcinoma (data not shown). Since no significant correlation was observed between any pairs of these antigens, combination assays should be of practical value in screening and monitoring the results of treatment.

As shown in Fig. 3, the combination of these 3 antigens in all lung cancer was positive in $68 \%$ of cases. Seventy-three percent of pulmonary adenocarcinoma pleural effusions were positive for the combination assay using SLEX and SLX, $77 \%$ for the SLEX and CA19-9 combination, and 68\% for the SLX and CA199 combination. Eighty-three percent of effusions were positive for at least one of these 3 antigens. Of the benign effusions, only $6 \%$ were positive for at least one
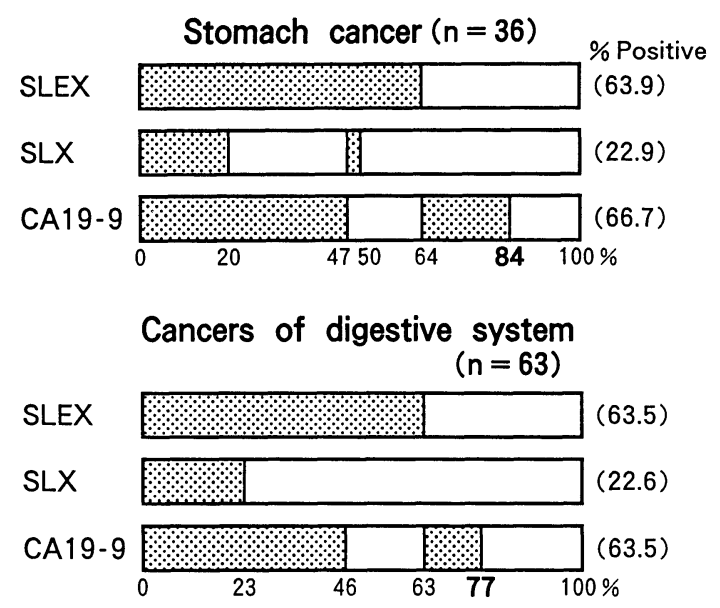

Cancers other than digestive system

$(n=16)$

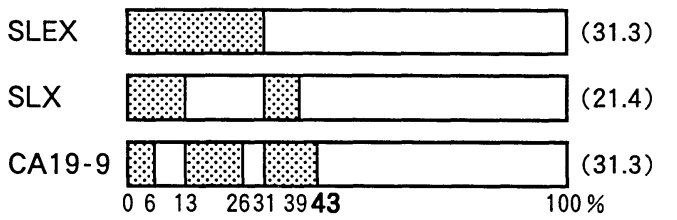

Fig. 4. The combination assay of the three antigens in the fluid samples from 63 patients with cancer of digestive system including 36 gastric cancers and 16 patients with other types of cancer. Numbers in parentheses on right side indicate positivity rates in each antigen. Boldface numbers indicate the total percentages of which at least one of three antigens is positive in each group. 
of the 3 antigens (Fig. 3).

Fig. 4 shows the results of combination assay using these 3 antigens in malignant fluid specimens from cancers other than lung cancer. As shown in the upper and middle panels, SLX-positive cases were included among the SLEXpositive ones in digestive organ cancers. Therefore, the combination assay for such malignancies was performed using only SLEX and CA19-9. Seventy-seven percent of the 63 digestive organ cancer fluids specimens were positive for at least one of these 2 antigens, including $84 \%$ of gastric carcinoma. The combination assay for malignant fluid specimens from cancers other than digestive organ cancers, was positive for at least 1 of the 3 antigens in $43 \%$ of cases (lower panel of Fig. 4).

\section{Fractionation of the malignant fluid specimens}

Fractionation of pleural effusions from lung cancer patients on a Sephacryl S-300 column revealed that SLEX antigen was eluted in the void volume identical
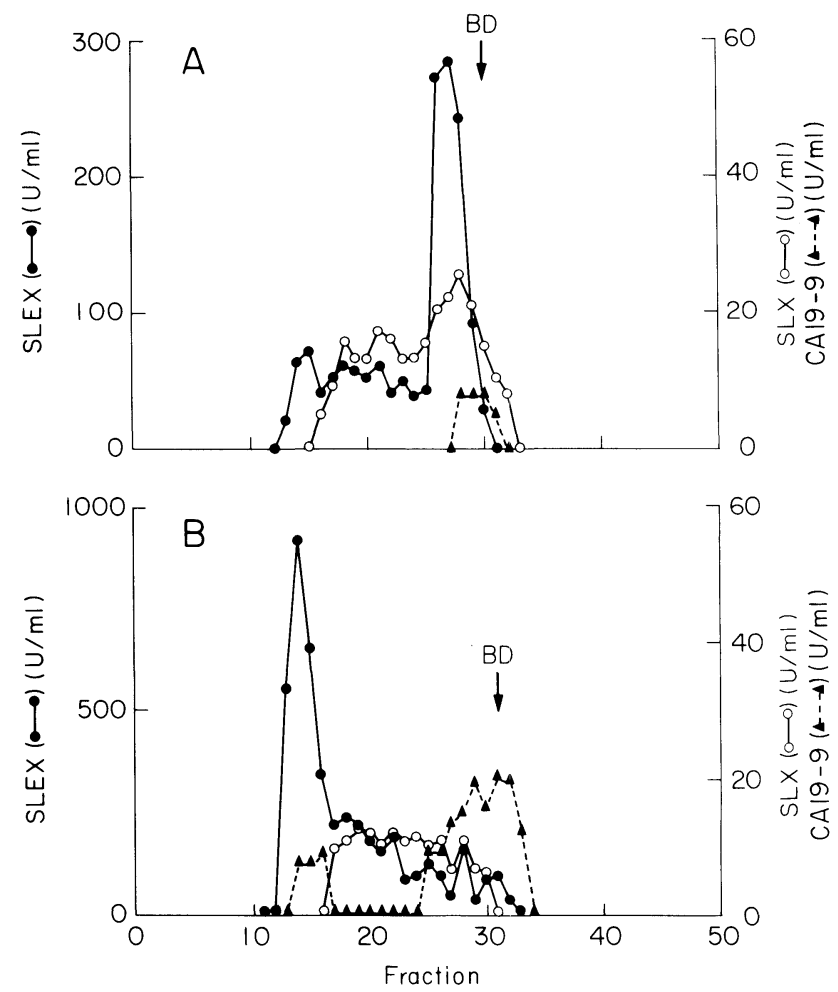

Fig. 5. Gel chromatography of malignant pleural effusions from patients with pulmonary adenocarcinoma (A) and with colon cancer (B) using Sephacryl S-1000. Fractions were monitored for $\operatorname{SLEX}(\bullet)$ by FEIA, for SLX $(\bigcirc-0)$ by RIA, and for CA19-9 (_- ) by RIA as described in "Materials and Methods". BD indicates the position where blue dextran was eluted. 


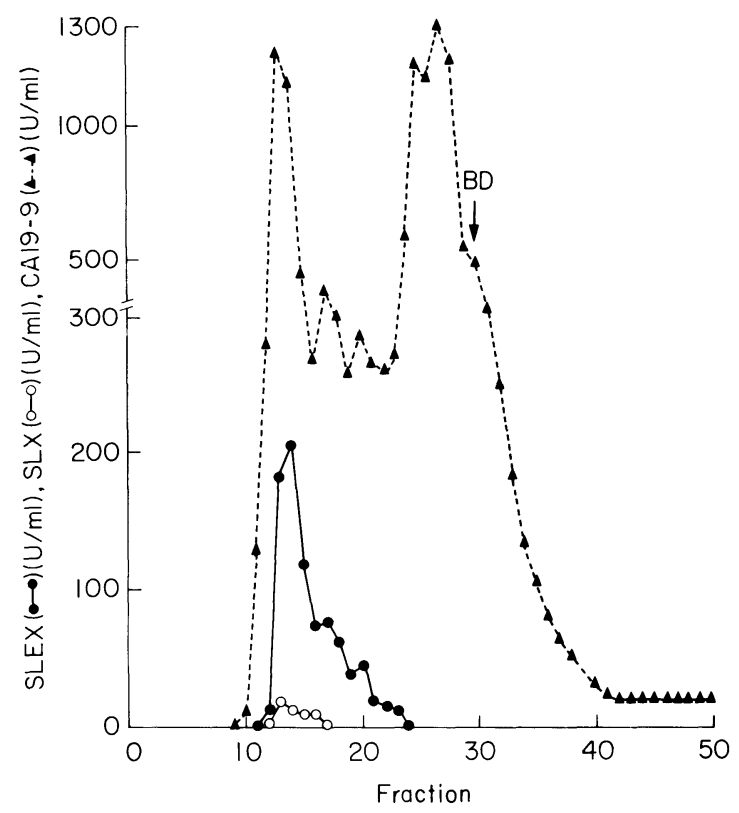

Fig. 6. Gel chromatography of the bile from patient with bile duct cancer using Sephacryl S-1000. Fractions were monitored for SLEX $(\bullet \bullet)$ by FEIA, for SLX $(\bigcirc-\circ)$ by RIA, and for CA19-9 ( $--\Lambda)$ by RIA as described in "Materials and Methods". BD indicates the position where blue dextran was eluted.

with the peak of high molecular weight sialic acid, indicating a sialic acidcontaining compound (data not shown). Fig. 5 shows the results of fractionation of pleural effusions from patients with pulmonary adenocarcinoma (A) or colon cancer (B) on a Sephacryl S-1000 column using blue dextran 2000 as a position indicator.

The SLEX antigen peaks in panels (A) and (B) were shifted to the left in the void volume from the position of blue dextran 2000 , indicating a molecular weight of $>2 \times 10^{6}$. The elution patterns for CA19-9 from the same pleural effusions were essentially the same as those of SLEX, although an other peak was also observed at the position indicated by blue dextran 2000, as shown in the lower panel (B). SLX was shown to elute broadly without a definite peak in the pleural fluid from patients with types of cancer (Fig. 5).

Gel chromatography using Sephacryl S-1000 of bile from a bile duct cancer patient (Fig. 6) showed that SLEX and CA19-9 were eluted at the same position as seen in the pleural effusions from colon cancer patients in Fig. 5. The titer of CA19-9 antigen was, however, greater than that shown in Fig. 5. SLX was also eluted in the void volume with a single peak at quite a low. 


\section{Discussion}

The detection of soluble tumor antigens in serous fluids may offer an approach to the diagnosis of carcinoma independent of cytological examination. CEA has been utilized most widely as a tumor marker for serous effusions (Nystrom et al. 1977 ; Whiteside and Dekker 1979; Vladutiu et al. 1979, 1981 ; Niwa et al. 1985 ; Tamura et al. 1988). In the present study, we demonstrated that the SLEX antigen could be the most useful tumor marker among the three carbohydrate antigens tested in patients with various types of cancer, and especially in pulmonary adenocarcinoma. These three antigens were present at high levels in the pleural effusions of patients with adenocarcinoma of the lung, with SLEX showing the highest level of positivity among the three antigens tested (as it also did for all lung cancer). Although the number of patients with pleural effusions due to lung cancers other than adenocarcinoma was small, impressive differences were noted between either the antigen titers or the positivity rates in adenocarcinoma and the other types of lung cancer (Fig. 1). The finding regarding SLEX and SLX was similar to that seen in analysis of serum samples from patients with pulmonary adenocarcinoma (Hirota et al. 1985a, b ; Kannagi et al. 1986 ; Zenita et al. 1988), and corresponds well with the immunohistochemical finding that SLEX antigen is maximally expressed in this type of adenocarcinoma, and that its staining intensity is significantly stronger (Hirota et al. 1986). It also corresponds with the finding that SLX antigen was also observed in well and moderately differentiated adenocarcinoma of the lung, but less often in poorly differentiated adenocarcinoma, large cell carcinoma, squamous cell carcinoma, and small cell carcinoma (Miyake et al. 1988). Since SLEX was detected more commonly than SLX even though these two antigens both belong to the group of sialylated type 2 chain antigen, SLEX appears to be more sensitive and specific for cancer than SLX.

Sialylated Le ${ }^{a}$ is recognized by CSLEA1 (Galton et al. 1985) which is similar but not identical to NS19-9 (Magnani et al. 1982). This antigen was detected in the serum of patients with lung cancer, predominantly adenocarcinoma (Hirota et al. 1985b), and was shown to be expressed in lung cancer by immunohistochemical studies, with expression being greater in adenocarcinoma than in squamous, large cell, or small cell carcinoma (Kasai et al. 1986). CA19-9 belongs to the same group of sialylated type 1 chain antigens and it was thus thought that it may also be recognized in pulmonary adenocarcinoma by NS19-9 (Magnani et al. 1982), although it has been mainly shown to be present in the serum of patients with gastrointestinal and pancreatic cancer (Herlyn et al. 1982). However, although elevated levels of CA19-9 were found in the pleural effusions of patients with pulmonary adenocarcinoma (Fig. 1), the positivity rate was lower than that for SLEX. This may perhaps be explained by the difference in expression observed immunohistochemically between SLEX and CA19-9 (unpublished data). 
SLEX could be more useful than the other antigens even in assessing the malignant fluid samples of digestive cancers. SLEX had the same positivity rate as CA19-9 (63.5\% of all digestive cancers), and detected $75 \%$ of bile duct cancers and $100 \%$ of colorectal cancers (Fig. 2). In contrast, SLX had much lower positivity rates than the other two antigens. Although SLEX positivity was a little lower than that of CA19-9 in patients with gastric and pancreatic cancer, SLEX appears to be more specific for malignancy than is the other antigen because it has been reported that normal tissue distribution differs between SLEX and CA19-9. SLEX is not detected in the normal stomach, bile ducts, and pancreatic ducts (Fukushima et al. 1984), while CA19-9 normally is expressed in these tissues (Atkinson et al. 1982).

Since no correlation was seen between any pairs of the three antigens, the detection of tumor-associated antigens can be increased by a combination assay using these 3 antigens. Combination assay increased the positivity rate markedly to as high as $68 \%$ in lung cancer, including $83 \%$ of adenocarcinoma (Fig. 3 ). Since all of cases with positive SLX were also positive for both SLEX and CA199 in patients with digestive cancer, the combination of these 2 antigens was sufficient for detecting such cancers. For example, in gastric carcinoma $84 \%$ of the fluid samples were positive for at least 1 of these 2 antigens (Fig. 4). We emphasize that the combination of these two antigens was especially useful because they belong to different types of blood group-associated antigens.

Tumor-associated carbohydrate antigens, including CA19-9, have been shown to be carried on high molecular weight glycoproteins in the serum (Magnani et al. 1982, 1983 ; Hirohashi et al. 1984; Kannagi et al. 1988). Based on the data presented in this paper, it appears that the three antigens possibly exist on molecules with a high molecular weight more than $2 \times 10^{6}$ in malignant fluid as well as in the serum. The activities of these three antigen were eluted mostly at same fractions. In addition the activity detected by the double determinant assay with NS19-9 for cather and CSLEX1 for tracer coincided with that of CSLEX1 for cather and NS19-9 for tracer, and the peaks of both activities existed mostly in the void volume of protein (data not shown), suggesting that the carriers of SLEX, CA19-9 and SLX in pleural effusion may be the same molecules. However the existence pattern of the antigens in malignant fluid remains obscure and should be evaluated in the future. For the purpose of distinguishing between benign and malignant effusions, further investigations, (e.q., the correlation between the SLEX level and the number of cancer cells) will be neccessary using fluid samples which are not defined cytologically. Since the tumor-associated antigen SLEX was a more useful tumor marker than either SLX or CA19-9, and since combination assay resulted in an increased rate of detection of cancer, both assays for SLEX and combination assays have potential applications in monitoring the response of various cancers to treatment. 


\section{Acknowledgments}

I thank Drs. Kiyoyasu Fukushima, Kazuhito Hiratani and Masaki Hirota for their helpful advises and consultation, and Mr. Atsushi Yokoyama for his technical assistance, and Kohei Hara, M.D. for his review.

\section{References}

1) Atkinson, B., Ernst, C., Heylyn, M., Stepleoski, Z., Sears, H.F. \& Koplowski, H. (1982) Gastro-intestinal cancer-associated antigen in immunoperoxidase assay. Cancer Res., 42, 4820-4823.

2) Bast, R.C., Feeney, M., Lazarus, H., Nadler, L.M., Colvin, R.B. \& Knapp, R.C. (1981) Reactivity of a monoclonal antibody with human ovarian carcinoma. J. Clin. Invest., 68, 1331-1337.

3) Bast, R.C., Klug, T.L., John, E.S., Jenison, E., Niloff, J.M., Lazarus, H., Berkowitz, R.S., Leavitt, T., Griffiths, C.T., Parker, L., Zurawski, V.R. \& Knapp, R.C. (1983) A radioimmunoassay using a monoclonal antibody to monitor the course of epitherial ovarian cancer. N. Engl. J. Med., 309, 883-887.

4) Fukushima, K., Hirota, M., Terasaki, P.I., Wakisaka, A., Togashi, H., Chia, D., Suyama, N., Fukushi, Y., Nudelman, E. \& Hakomori, S. (1984) Characterization of sialosylated Lewis ${ }^{\times}$as a new tumor-associated antigen. Cancer Res., 44, 5279-5285.

5) Fuziwara, K., Matsumoto, N., Yagisawa, S., Tanimori, H., Kitagawa, T., Hirota, M., Hiratani, K., Fukushima, K., Tomonaga, A., Hara, K. \& Yamamoto, K. (1988) Sandwich enzyme immunoassay of tumor-associated antigen sialosylated Lewis ${ }^{\times}$using $\beta$-D-galactosidase coupled to a monoclonal antibody IgM isotype. J. Immunol. Methods, 112, 77-83.

6) Galton, J., Terasaki, P.I., Wakisaka, A., Chia, D., Katz, D. \& Hardiwidjaja, S. (1985) A monoclonal antibody reactive with colon, stomach and pancreatic adenocarcinomas. In: Milgrom, Abeyounis, Albini (eds.), Antibodies: Protective, Destructive and Regulatory Role. Ninth International Convocation of Immunology, Buffalo-New York-Basel-Karger, pp. 117-125.

7) Herlyn, M., Sears, H.F., Steplewski, Z. \& Koprowski, H. (1982) Monoclonal antibody detection of a circulating tumor-associated antigen: I. Presence of antigen in sera of patients with colorectal, gastric, and pancreatic carcinoma. J. Clin. Immunol., 2, $135-140$.

8) Hirohashi, S., Watanabe, M., Shimosato, Y. \& Sekine, T. (1984) Monoclonal antibody reactive with the sialylo-sugar residue of a high molecular weight glycoprotein in sera of cancer patients. Gann, 75, 485-488.

9) Hirota, M., Fukushima, K., Terasaki, P.I., Terashita, G.Y., Kawahara, M., Chia, D., Suyama, N. \& Togashi, H. (1985a) Sialosylated Lewis ${ }^{\times}$in the sera of cancer patients detected by a cell-binding inhibition assay. Cancer Res., 45, 1901-1905.

10) Hirota, M., Fukushima, K., Terasaki, P.I., Terashita, G.Y., Galton, J. \& Kawahara, M. (1985b) Detection of tumor-associated antigens in the sera of lung cancer patients by three monoclonal antibodies. Cancer Res., 45, 6453-6456.

11) Hirota, M., Fukushima, K., Hiratani, K., Kadota, J., Nakashima, M., Tomonaga, A., Yokoyama, A. \& Hara, K. (1986) Tumor-associated carbohydrate antigens: Lewis ${ }^{\times}$ and sialylated Lewis ${ }^{\times}$in primary lung adenocarcinoma. Med. Biol., 113, 403-405.

12) Kannagi, R., Fukushi, Y., Tachikawa, T., Noda, A., Shin, S., Shigeta, K., Hiraiwa, N., Fukuda, Y., Inamoto, T., Hakomori, S. \& Imura, H. (1986) Quantitative and qualitative characterization of human cancer-associated serum glycoprotein antigens expressing fucosyl or sialyl-fucosyl type 2 chain polylactosamine. Cancer Res., 46, $2619-2626$. 
13) Kannagi, R., Kitahara, A., Itai, S., Zenita, K., Shigeta, K., Tachikawa, T., Noda, A., Hirano, H., Abe, M., Shin, S., Fukushi, Y., Hakomori, S. \& Imura, H. (1988) Quantitative and qualitative characterization of human cancer-associated serum glycoprotein antigens expressing of sialyl or sialyl-fucosyl type 1 chain. Cancer Res., 48, 3856-3863.

14) Kasai, K., Kameya, T., Okuda, T., Terasaki, P.I. \& Iwaki, Y. (1986) Immunohistochemical examination of lung cancers using monoclonal antibodies reacting with sialosylated Lewis ${ }^{\times}$and sialosylated Lewis ${ }^{\mathrm{a}}$. Virchows Arch. A, 410, 253-261.

15) Magnani, J.L., Nilsson, B., Brockhaus, M., Zopf, D., Steplewski, Z., Koprowski, H. \& Ginsburg, V. (1982) A monoclonal antibody-defined antigen associated with gastrointestinal cancer is a ganglioside containing sialylated lacto-N-fucopentaose II. $J$. Biol. Chem., 257, 14365-14369.

16) Magnani, J.L., Steplewski, Z., Koprowski, H. \& Ginsburg, V. (1983) Identification of the gastrointestinal and pancreatic cancer-associated antigen detected by monoclonal antibody 19-9 in the sera of patients as a mucin. Cancer Res., 43, 5489-5492.

17) Metzgar, R.S., Rodriguez, N., Finn, O.J. Lan, M.S., Daasch, V.N., Fernsten, P.D., Meyers, W.C., Sindelar, W.F., Sandler, R.S. \& Seigler, H.F. (1984) Detection of a pancreatic cancer-associated antigen (DU-PAN-2 antigen) in serum and ascites of patients with adenocarcinoma. Proc. Natl. Acad. Sci. USA, 81, 5242-5246.

18) Miyake, M., Zenita, K., Tanaka, O., Okada, Y. \& Kannagi, R. (1988) Stage-specific expression of SSEA-1-related antigens in the developing lung of human embryos and its relation to the distribution of these antigens in lung cancers. Cancer Res., 48, $7150-7158$.

19) Niwa, Y., Kishimoto, H. \& Shimokata, K. (1985) Carcinomatous and tuberculous pleural effusions : Comparison of tumor markers. Chest, 87, 351-355.

20) Nystrom, J.S., Dyce, B., Wada, J., Bateman, J.R. \& Havarback, B. (1977) Carcinoembryonic antigen titers on effusion fluid: A diagnostic tool? Arch. Intern. Med., 137, 875-879.

21) Tamura, S., Nishigaki, T., Moriwaki, Y., Fujioka, H., Nakano, T., Fujii, J., Yamamoto, T., Nabeshima, K., Noda, T. \& Higashino, K. (1988) Tumor markers in pleural effusion diagnosis. Cancer, 61, 298-302.

22) Vladutiu, A.O., Adler, R.H. \& Brason, F.W. (1979) Diagnostic value of biochemical analysis of pleural effusions: Carcinoembryonic antigen and beta ${ }_{2}$-microglobulin. Am. J. Clin. Pathol., 71, 210-214.

23) Vladutiu, A.O., Brason, F.W. \& Adler, R.H. (1981) Differential diagnosis of pleural effusions: Clinical usefulness of cell marker quantitation. Chest, 79, 297-301.

24) Whiteside, T.L. \& Dekker, A. (1979) Diagnostic significance of carcinoembryonic antigen levels in serous effusions : Correlation with cytology. Acta Cytol., 23, 443448.

25) Zenita, K., Kirihata, Y., Kitahara, A., Shigeta, K., Higuchi, K., Hirashima, K., Murachi, T., Miyake, M., Takeda, T. \& Kannagi, R. (1988) Fucosylated type 2 chain polylactosamine antigens in human lung cancer. Int. J. Cancer, 41, 344-349. 